\title{
Modelling the Effects of Conductive Polymers and Stem Cells Derived Myocytes on Scarred Heart Tissue
}

\author{
Damiano Fassina ${ }^{1}$, Caroline Mendonca Costa $^{2}$, Stefano Longobardi ${ }^{2}$, Sian E Harding ${ }^{1}$, Steven A \\ Niederer $^{2}$ \\ ${ }^{1}$ National Heart and Lung Institute, Imperial College London, UK \\ ${ }^{2}$ School of Biomedical Engineering and Imaging Sciences, King's College London, UK
}

\begin{abstract}
Myocardial infarction leads to permanent tissue scars that impairs cardiac function and can result in heart failure. The use of engineered heart tissue (EHT) and conductive polymers $(C P)$ in regenerative medicine aims to assist tissue recovery and improve of cardiac function. However, the attachment of EHT to the epicardium may disrupt tissue electrophysiology and lead to an increased arrhythmia risk.

We investigated the role that material properties, i.e. EHT and CP conductivity and thickness and EHT-tissue contact area, play in cardiac recovery, in the presence of scars with different depth, length and conductivity.

We created a $2 D$ model to simulate EHT and CP placed over a scar in rabbit ventricle. We performed a Global Sensitivity Analysis (GSA) to identify which parameters had the largest impact on electrical propagation across the scar and the EHT.

Our model showed that in case of non-transmural scar the main determinants were scar depth and conductivity, explaining $30 \%$ and $33 \%$ of the variance, respectively. However, in case of transmural scar, the model indicated the EHT conductivity and the extent of the EHT-tissue contact explain $40 \%$ and $46 \%$ of the variance, respectively.
\end{abstract}

\section{Introduction}

Myocardial infarction (MI) hospitalizes 1 person every 5 minutes in the UK [1]. Although 7 persons out of 10 survive [2], their heart can be permanently compromised.

MI ultimately results in permanent scar tissue. The presence of scar tissue in the heart can have a severe impact on cardiac function, exposing patients to increased risk of arrhythmias and heart failure.

Currently, cardiac function can only be restored with cardiac transplantation. However, cardiac regenerative medicine has recently been proposed as a promising alternative. It comprises a wide spectrum of novel treatments, which aim to replace or augment the function of tissue lost to cardiac infarcts. In this work we focus on the creation and application of patches of cardiomyocytes derived from human induced pluripotent stem cells (hiPSC-CM) over the infarct.

HiPSC-CM are usually delivered in combination with scaffolds, due to their low (1-5\%) retention rate [3]. Desirable features for a scaffold used in cardiac engineering include biocompatibility, electrical conductivity and elasticity similar to the native myocardium [4]. Although many different kinds of scaffolds have been tested, we focus on the use of scaffolds manufactured with conductive polymers, since contraction and relaxation of the heart depends on the conduction of electrical impulses.

The patch-recipient heart interaction plays a crucial role in determining the success of the treatment with EHT. The hiPSC-CM may introduce electrical heterogeneity in the recipient heart, with areas of slow conduction and longer/shorter action potential duration and cause ectopic beats [5]. These can in turn lead to the initiation of life-threatening arrhythmias.

Potential arrhythmic risk of the patch can be mitigated by altering hiPSC-CM properties pharmacologically, or by altering the properties of the patch scaffolding [6]. However, the time and cost of experimentally optimising this combination of factors remain prohibitive. Modelling in silico the physiological processes of the heart and its interaction with the EHT and CP might address this bottleneck.

In this work we developed a 2D model of scarred rabbit myocardial tissue in the presence of a patch consisting of EHT and CP. The model allows us to simulate electrical propagation through the tissue and to determine the propagation pathway and the occurrence of conduction block. This provides an in silico environment where we can assess how impulse propagation is affected by variations of EHT's and CP's physical properties. To analyse the model we performed a global sensitivity analysis (GSA) to identify which model parameters have the largest impact on stimulus propagation. 


\section{Methods}

\subsection{Model geometry and parameters}

We created a 2D model representing a section of a scarred slab of rabbit tissue, with a patch bridging the two ends of healthy myocardium on either side of the scar (Figure 1). The patch is modelled as a layer of hiPSC$\mathrm{CM}$, which we refer to as EHT for brevity, combined with a layer of CP. The model was designed with 10 input parameters: tissue thickness (3.6-4.4 mm), internal bath thickness (0.1-1 mm), EHT thickness (0.1-2 mm) and conductivity (0.028 $-0.22 \mathrm{~S} / \mathrm{m}), \mathrm{CP}$ thickness $(0.5-2 \mathrm{~mm})$ and conductivity $(0-18 \mathrm{~S} / \mathrm{m})$, scar radius $(0.5-4 \mathrm{~mm})$, depth (10-100\% of tissue thickness) and conductivity (0$0.14 \mathrm{~S} / \mathrm{m})$, thickness of the EHT-slab tissue contact area (0.1-2 mm). Each parameter was varied independently across the range indicated in the brackets.

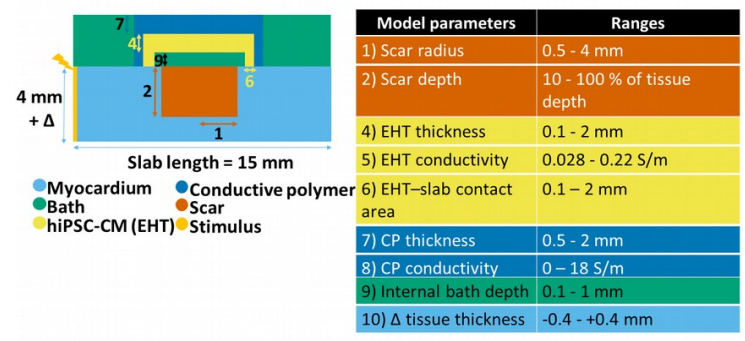

Figure 1. Schematic representation of the model. Myocardium, scar, EHT, CP and bath are represented in light blue, red, yellow, dark blue and green, respectively.

\subsection{Creation of 5 data set}

We considered 5 set ups. First, we considered the full model, with all 10 parameters, and we labelled it as epiendo, because the scar grows from epicardium to endocardium. The $2^{\text {nd }}$ set was obtained considering the same 10-dimensional parameters space of the $1^{\text {st }}$ set, but with the scar expanding from endocardium to epicardium in the depth dimension, and it was called endo-epi. The $3^{\text {rd }}$ set, called transmural, was obtained fixing the depth parameter of the scar, forcing the scar to be always transmural. The $4^{\text {th }}$ set was created fixing the conductivity of the scar to $0 \mathrm{~S} / \mathrm{m}$, as well as the depth. This set was called block. Finally, the $5^{\text {th }}$ set was formed by fixing also the radius of the scar (radius fixed), effectively fixing all of the 3 parameters relative to the scar and reducing the parameters to 7 . For each scenario we sampled the ndimensional parameters space using a Latin hypercube to obtain 5 sets of 1000 different parameters combinations.

Propagation of the cardiac action potential was simulated for each of the 5 sets, for each of the 1000 parameters combinations, resulting in 5000 tissue simulations.

\subsection{Simulating the electrophysiology}

Propagation of the action potential was simulated using the bidomain model [7]. This model was coupled with two different cellular-level electrophysiology models, Paci2018 [8] for the hiPSC-CM and UCLA_RAB [9] for the rabbit healthy tissue. The CP is modelled as an ohmic conductor [10]. The models' equations were solved using the Cardiac Arrhythmia Research Package (CARP) [11]. The tissue is stimulated with extracellular current injection on the left side of the slab, across the whole slab section.

\subsection{Determining the stimulus path}

For each simulation the activation times were computed as the time where the membrane voltage reaches a threshold of $-10 \mathrm{mV}$. These were computed at 8 assigned points of the slab to investigate whether the stimulus reaches the slab's top right corner, and the quickest path undertaken by the stimulus to reach this location. This enabled us to associate parameter ranges with each path.

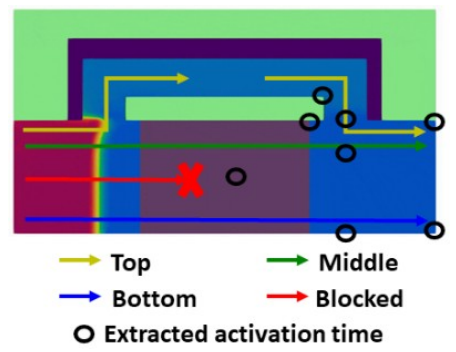

Figure 2. Screenshot of the stimulus propagation in one of the simulations. The arrows show which paths are linked to each of the 4 categories.

\subsection{Gaussian Processes emulator and Global Sensitivity Analysis}

In order to perform a systematic investigation on the importance of each model parameter, we fitted a Gaussian process emulator $[12,13]$ to our model, enabling us to perform a GSA. GSA's aims to quantify the relative importance of input variables in determining the value of an assigned output variable [14], which in our model is represented as activation times, collected on the top right corner of the tissue slab. Therefore, this procedure allows us to infer how each input parameter affects the activation times. 


\section{Results}

\subsection{Relevant parameters regions}

The analysis of the extracted activation times allows us to classify each of the 1000 simulation according to which patch the stimulus has followed. Figure 3 shows how sets 3 (block) and 4 (radius fixed) have the highest numbers of blocked cases, i.e. cases in which the stimulus does not reach the other side of the slab.
EHT-slab contact area below $0.8 \mathrm{~mm}$ the stimulus is always blocked. More than $95 \%$ of the blocked cases lay in the area defined by the aforementioned values of the two parameters.

\subsection{Sensitivity indexes}

Total effect (TE) indexes indicate each parameter's contribution to the output variance, and they have to be $<=1$.

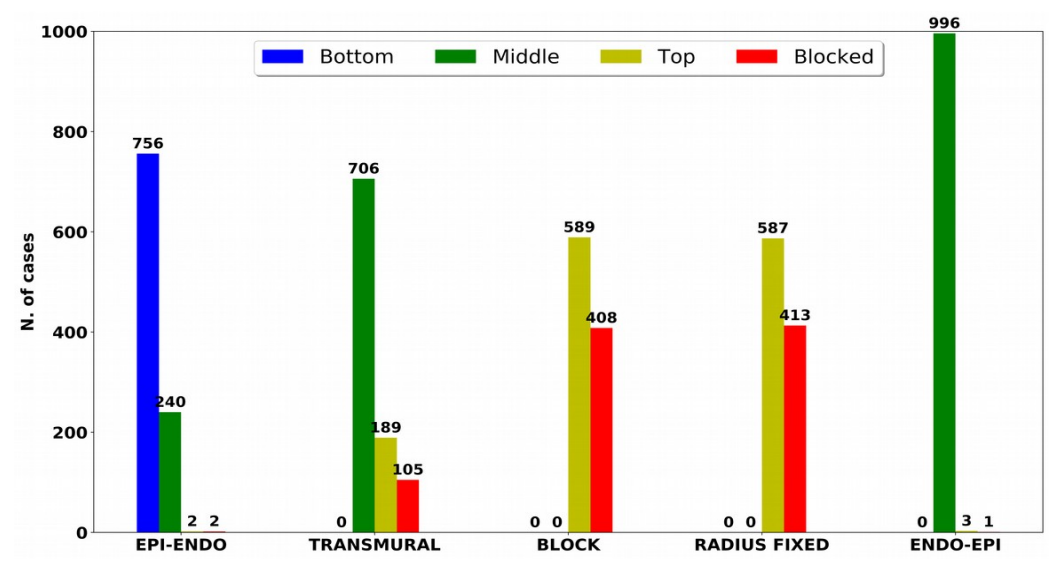

Figure 3. Distributions of the stimulus path in the 5 different sets of simulations

Figure 4 shows set 3's 8-dimensional parameters space, visualized by plotting the 8 parameters against each other. Each dot is a value assumed by the parameter in at least one of the simulations and is colored based on which path the stimulus has followed in that specific simulation. Red dots are overlaid on yellow dots. Panel B) highlights the plot of EHT conductivity against EHTslab contact area. The simulations indicate that for values of EHT conductivity below $0.07 \mathrm{~S} / \mathrm{m}$ and for values of
The first set of simulations showed that the stimulus propagation was mainly affected by the scar parameters. Scar conductivity and depth scored 0.46 and 0.41 respectively. However, the simulations in which the scar parameters were fixed demonstrated the importance of the EHT conductivity $(\mathrm{TE}=0.61)$ and the EHT-slab contact area $(\mathrm{TE}=0.69)$. These results confirmed the importance of the relation between the EHT conductivity and the thickness of the EHT-slab tissue contact area, as highlighted during the stimulus path investigation (Figure 4).

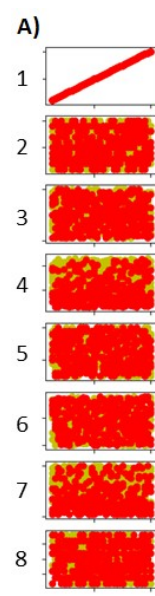

1

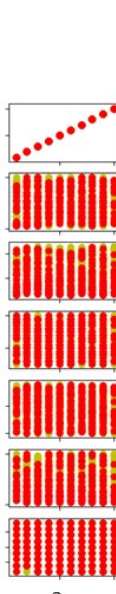

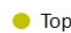

Top

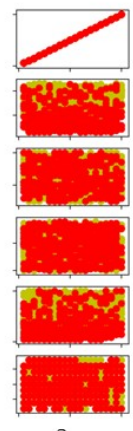

- Blocked

B)

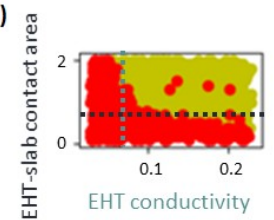

Figure 4. A) Parameters of the 8-dimensional space plotted against each other. 1: scar radius, 2: internal bath thickness, 3: EHT thickness, 4: EHT conductivity, 5: CP thickness, 6: CP conductivity, 7: EHT-tissue slab contact area, 8: $\Delta$ tissue thickness. B) Dashed lines highlight the thresholds below which the stimulus is always blocked. 
GSA - TOTAL EFFECT INDEX

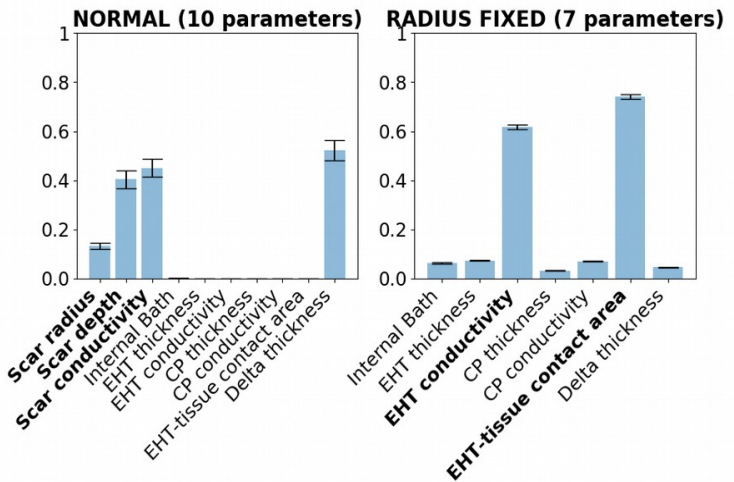

Figure 5. Total effect indexes with non-fixed scar parameters (left) and fixed scar parameters (right).

\section{Discussion}

Our model simulates electrical propagation in a slab of rabbit myocardium in the presence of a scar and an engineered patch formed by a combination of a hiPSCCM layer and a CP layer.

The simple geometry of the model allows for quick and computationally cheap simulations, enabling us to vary the physical properties of the patch materials, as well as the scar characteristics.

Our model showed that the physical properties of the materials attached to the epicardium do not have a considerable impact on the tissue electrophysiology in case of non-transmural scar. However, in case of transmural scar, the model indicated the EHT conductivity and the extent of the EHT-slab tissue contact area have an active role in influencing stimulus propagation. These observations are consistent with experimental findings, where an unsuccessful patchrecipient heart coupling has been linked to the limited extension of their contact area [15].

These in silico findings could guide future experimental work using hiPSC-CMs and CP patches.

\section{Acknowledgments}

This research project is funded by a British Heart Foundation, Centre of Regenerative Medicine - NHLI Studentship.

\section{References}

[1] UK hospital statistics, 2018-19.

[2] Smolina, K., Wright, F. L., Rayner, M., \& Goldacre, M. J., "Determinants of the decline in mortality from acute myocardial infarction in england between 2002 and 2010: linked national database study”, Bmj , 344 , d8059, 2012

[3] Chow, A., Stuckey, D. J., Kidher, E., Rocco, M., Jabbour,
R. J., Mansfield, C. A., Athanasiou, T., "Human induced pluripotent stem cell-derived cardiomyocyte encapsulating bioactive hydrogels improve rat heart function post myocardial infarction", Stem Cell Reports, 9 (5), 14151422, 2017.

[4] Maidhof, R., Tandon, N., Lee, E. J., Luo, J., Duan, Y., Yeager, K., Vunjak-Novakovic, G., "Biomimetic perfusion and electrical stimulation applied in concert improved the assembly of engineered cardiac tissue”, Journal of tissue engineering and regenerative medicine, 6 (10), e12-e23, 2012.

[5] Huang, K., Hu, S., \& Cheng, K., "A new era of cardiac cell therapy: Opportunities and challenges", Advanced Healthcare Materials, 1801011, 2018.

[6] Goßmann, M., Frotscher, R., Linder, P., Neumann, S., Bayer, R., Epple, M., Artmann, G. M., "Mechanopharmacological characterization of cardiomyocytes derived from human induced pluripotent stem cells", Cellular Physiology and Biochemistry, 38 (3), 1182-1198, 2016.

[7] Clayton, R., Bernus, O., Cherry, E., Dierckx, H., Fenton, F. H., Mirabella, L., Zhang, H., "Models of cardiac tissue electrophysiology: progress, challenges and open questions", Progress in biophysics and molecular biology, 104 (1-3), 22-48, 2011.

[8] Paci M, Polonen R. P, Cori D, Penttinen K, Aalto-Setala K, Severi S, Hyttinen J, "Automatic optimization of an in silico model of human iPSC derived cardiomyocytes recapitulating calcium handling abnormalities", Frontiers in physiology, 9, 709, 2018.

[9] Mahajan A, Shiferaw Y, Sato D, et al, “A rabbit ventricular action potential model replicating cardiac dynamics at rapid heart rates”, Biophysical journal, vol. 94,2: 392-410, 2008.

[10] Stavrinidou, E., Leleux, P., Rajaona, H., Fiocchi, M., Sanaur, S., \& Malliaras, G. G., “A simple model for ion injection and transport in conducting polymers", Journal of Applied Physics, 113 (24), 244501, 2013.

[11] Vigmond EJ, Weber dos Santos R, Prassl AJ, Deo M, Plank G., "Solvers for the cardiac bidomain equations", Progress in Biophysics and Molecular Biology, 96(1-3):3-18, 2008.

[12] Rasmussen, C. E, "Gaussian processes in machine learning. In Summer school on machine learning” (pp. 63-71), 2003.

[13] Longobardi, Stefano, et al. "Predicting left ventricular contractile function via Gaussian process emulation in aortic-banded rats.", Philosophical Transactions of the Royal Society A, 378.2173, 2020.

[14] Sobol, I. M, "Global sensitivity indices for nonlinear mathematical models and their montecarlo estimates", Mathematics and computers in simulation, 55 (1-3), 271280, 2001.

[15] Jackman, Christopher P et al., "Engineered cardiac tissue patch maintains structural and electrical properties after epicardial implantation”, Biomaterials, vol. 159: 48-58, 2019.

Address for correspondence:

Damiano Fassina

National Heart and Lung Institute

Imperial College London

United Kingdom

d.fassina18@imperial.ac.uk 